\title{
Classical analysis of a network model of quantum Hall systems
}

\author{
K. I. Wysokiński, ${ }^{*}$ F. Evers, ${ }^{\dagger}$ and W. Brenig \\ Physik Department T-30, Technische Universität München, J. Franck Strasse 1, 85748 Garching, Federal Republic of Germany
}

(Received 28 June 1995)

\begin{abstract}
A version of a network model of quantum Hall systems is studied classically. We assume that randomness inherent in the problem enters the model via random heights of saddle points only. We use ideas from classical percolation theory to calculate numerically the fractal dimension $d_{f}$, the correlation length exponent $\nu$, the diffusion coefficient $D$, the corresponding exponent $k$, and other parameters of interest. The width of the longitudinal conductivity peak scales with the classical localization length exponent $\nu$.

[S0163-1829(96)01936-4]
\end{abstract}

\section{INTRODUCTION}

Even though it has always been argued $^{1,2}$ that electronelectron interactions are not important for a description of the integral quantum Hall effect ${ }^{3}$ (QHE), it came as a surprise when experiments ${ }^{4,5}$ found the same value of the critical exponent for the delocalization transition as predicted by the single-particle calculations. ${ }^{6-9}$

It is interesting to note the agreement of the value of the localization length exponent $\nu=2.34 \pm 0.04$ obtained numerically for the lowest Landau level and uncorrelated disorder ${ }^{7}$ with one calculated analytically via semiclassical $\operatorname{arguments}^{8,10} \quad \nu=\frac{7}{3}$ and those obtained numerically from transfer-matrix analysis of the network models ${ }^{9}$ $\nu=2.5 \pm 0.5$ and $^{11} \nu=2.4 \pm 0.2$, which should apply to smoothly varying potentials and strong magnetic fields.

Moreover, in semiclassical approximations ${ }^{8,10}$ the wave interference effects (backscattering) seem to play only a minor role, and tunneling alone is made responsible for the change of the classical percolation exponent $\nu_{2 \mathrm{D}}=\frac{4}{3}$ to $\nu=1+\nu_{2 \mathrm{D}}=\frac{7}{3}$. The present understanding of the network models is that both, quantum tunneling and interference effects, are important to give the proper value of the exponent.

The applicability of semiclassical (percolation) arguments to the description of the integer QHE relies on the fact that states of disordered two-dimensional electron gases placed in high perpendicular magnetic fields $\mathbf{B}$ are concentrated on a strip of width $l$ ( $l$ is the magnetic length) along equipotential lines of the smoothly varying potential. ${ }^{9,12}$

In this work we shall use a representation of quantum Hall systems by means of a square network. The bonds of the network correspond to the equipotentials along which the guiding centers of the electronic orbits drift. The sites or nodes of the network represent saddle points of the real system. The idea behind the introduction of the network model in this context was that most interesting differences between classical and quantum behavior are connected with the possibility of tunneling at shallow saddle points. ${ }^{9,13}$

It is our aim to study the network model classically, and obtain information relevant to the QHE. In particular we are interested in values of classical exponents near the (classical percolation) delocalization transition. We study large networks with up to $3000 \times 3000$ saddle points with randomly distributed heights. We neglect all other possible randomness in the system, in particular the random distances between saddle points. There are no wave aspects of particle motion in the model. In this respect our study is complementary to a previous classical analysis of quantum Hall effect. ${ }^{14}$

Previous work ${ }^{9,13}$ on network models of quantum Hall systems concentrated on quantum tunneling and interference effects. Randomness entered the model through random phases of the wave function describing electrons moving along bonds of the network. Using the classical approach we completely neglect interference effects and also tunneling. Randomness enters our model through random "heights" of the saddle points.

Moreover, extensive use of ideas from percolation theory ${ }^{15,16}$ allows us to calculate not only the correlation length exponent $\nu$, the diffusion exponent $k$, and fractal dimension $d_{f}$, but also the longitudinal conductivity of the biased system and its critical behavior.

In our studies we also tried to allow for tunneling, but excluding interfence effects. Classically the trajectory near a given saddle point always turns either right or left depending on the energy difference of the trajectory and the saddle point. When incorporating tunneling into the model, we calculated the tunneling probability ${ }^{17}$ in either direction. In practice the direction of the further motion of the particle was determined by choosing a random number and comparing it with corresponding probabilities. This simplest treatment of tunneling leads to an increase of the correlation length even beyond the expected value $\nu=\frac{7}{3}$.

The organization of the paper is as follows. In Sec. II we define the model, explain our approach, and present some of the results. In Sec. III we present the details connected with a calculation of the longitudinal conductivity of the system, and define the corresponding exponents. A summary of the results and their discussion is given in Sec. IV.

\section{NETWORK MODEL}

A weakly disordered two-dimensional electron gas, when placed in strong perpendicular magnetic fields, exhibits at low temperature the quantization of the Hall resistance $\rho_{x y}$ for a wide range of magnetic field values accompanied by the vanishing of the longitudinal resistance $\rho_{x x}$. The physics of the QHE at the plateaus is quite well understood. $^{1,2}$ 
The transition region between consecutive Hall plateaus, where $\rho_{x y}$ is not quantized and $\rho_{x x}$ takes on nonzero values, has attracted a lot of attention recently. In fact, the experiments $^{4,5}$ have shown that this transition is a continuous phase transition with an electron localization length being the only divergent length scale:

$$
\xi \propto\left|B-B_{c}\right|^{-\nu} \text { or } \xi \propto\left|E-E_{c}\right|^{-\nu},
$$

where $B_{c}\left(E_{c}\right)$ are critical values of the magnetic field (Fermi energy) at which the transition occurs.

Consider a system of noninteracting two-dimensional electrons moving in a slowly varying potential $V(\mathbf{r})$ and subject to a strong perpendicular magnetic field $\mathbf{B}$ described by the vector potential $\mathbf{A}(\mathbf{r})$. The corresponding Hamiltonian can be written in the form

$$
H=\frac{1}{2 m^{*}}(\mathbf{p}+e \mathbf{A})^{2}+V(\mathbf{r}),
$$

with $\mathbf{p}=-i \hbar \nabla$, and $e$ and $m^{*}$ denoting the electron charge and the effective mass, respectively. The random potential $V(\mathbf{r})$ is assumed to vary on a scale $\lambda$ much larger than the magnetic length $l\left(l^{2}=\hbar / e B\right)$. The semiclassical picture of the quantum Hall effect is valid, strictly speaking, in the $B \rightarrow \infty$ limit. In the considered limit of $\lambda \gg l$, the electron motion consists of a cyclotron motion superimposed on a slow drift perpendicular to the local electric field $\vec{E}$, which is given by $-\nabla V(\mathbf{r})$. The eigenstates of the system have a nonzero amplitude in a strip of width $l$ along the equipotential lines $V(\mathbf{r})=E_{p}$. The corresponding eigenenergies are given by the sum of the kinetic energy of the cyclotron motion $\left(n+\frac{1}{2}\right) \hbar \omega_{c}\left(\omega_{c}=e B / m^{*}\right.$ is the cyclotron frequency, $n=0,1,2 \ldots$ denotes consecutive Landau levels) and the potential energy $E_{p}$. In infinite systems all equipotentials will be closed at energies $E_{p} \neq E_{c} . E_{c}$ is the only energy at which infinite trajectories exist. The contours at energy $E_{p}$ will come close together near the saddle points of the potential.

In a very interesting paper, Chalker and Coddington ${ }^{9}$ proposed replacing the real system by a network with nodes representing saddle points and links joining them. In their analysis the randomness of the lengths of equipotential lines between neighboring saddle points has been simulated by the randomness of phase factors in transfer matrices, ${ }^{9}$ but the randomness of the heights of saddle points was neglected. Lee, Wang, and Kivelson ${ }^{11}$ generalized the model, and also took the randomness of the heights into account. They claim to see the crossover between the classical and quantum limits as a function of the width $W$ of the probability distribution of the heights of saddle points.

We assume the nodes of the network to form a twodimensional square lattice. In a classical approach it is not important that we neglect the randomness in the distances between saddle points. Their heights are assumed to be uniformly distributed over the region $E_{s} \in[-W / 2, W / 2]$. In actual calculations we have taken $W=1$. This means that the system percolates at $E_{c}=0$. The distance between neighboring saddle points is taken as the unit of length, and the time required to traverse it is taken as the time unit.

If we assume the magnetic field to be applied in the positive $z$ direction, then the electron at energy $E$ approaching a saddle point having energy (height) $E_{s}$ will turn left (right) if

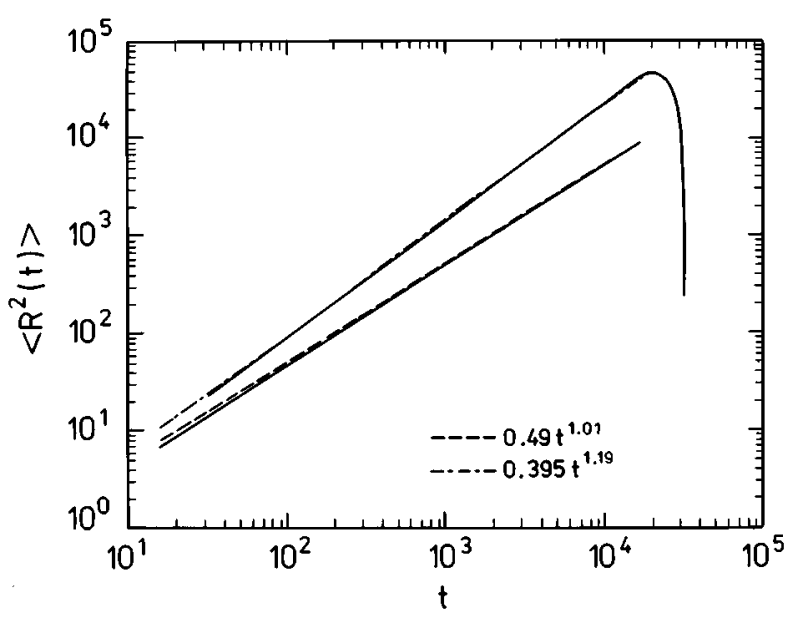

FIG. 1. The time dependence of the averaged extension of the trajectories $\left\langle R^{2}(t)\right\rangle$ at the percolation level. The thin full line and the dashed line refer to open trajectories. The dash-dotted line corresponds to averaging over trajectories spanning the $N \times M$ system.

$E-E_{s}>0(<0)$. It is the peculiarity of the system under study that near the saddle point the particle cannot move in a forward direction. As a consequence the trajectories of electrons in a network model have a very complicated shape.

Looking at the time dependence of the average extension $\left\langle R^{2}(t)\right\rangle$ of the region visited by the particle in a large $N \times M$ network, we can determine the diffusion exponent $k$, defined for large times $t$ through

$$
\left\langle R^{2}(t)\right\rangle=D t^{k}
$$

where $D$ is the diffusion coefficient. We considered the value of $k$ when only closed trajectories were taken into account, and obtained $k \approx 1$, indicating that at large times the diffusion is normal. When taking only open (i.e., those extended from one side of the network to the opposite one) trajectories into account, we obtained $k=1.20 \pm 0.05$. Both values coincide with those found in a previous classical study of quantum Hall systems. ${ }^{14}$ Our data obtained for systems with length $N=1000$ and width $M=500$ are shown in Fig. 1. We have averaged over 4000 realizations of saddle-point energy distributions.

It is the peculiarity of the system studied that localized states exist at every energy, even at the percolation level where also extended states exist. The picture of equipotential lines in random potentials is very helpful in understanding that. In the network representation the situation is quite the same. So if we look at possible trajectories of the particle in the network, we can ask what is the relation between their average extension $\langle R\rangle$ and their average length $\langle L\rangle$,

$$
\langle L\rangle=\operatorname{const}\langle R\rangle^{d_{f}}
$$

which defines the fractal dimension of the walk. We have found $d_{f}=1.7 \pm 0.05$ for both localized and extended trajectories. This value is compatible with the expected exact value $7 / 4$. Our ' 'random walker', on a network in fact probes what is called the "external hull." 15 The fractal dimension for a two-dimensional system at the percolation threshold is expected to be $91 / 48$ exactly. 


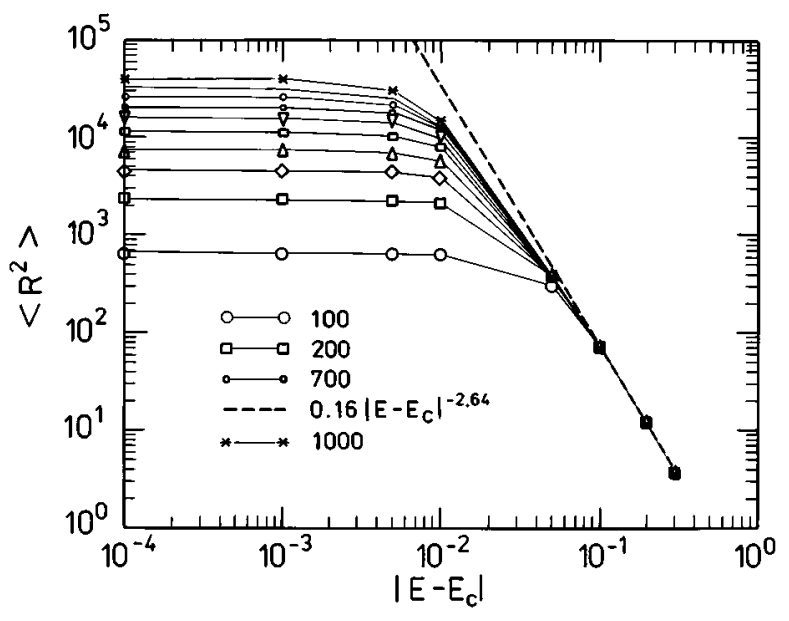

FIG. 2. The averaged square of the extension of electron trajectories vs energy relative to percolation level, and for a number of sample sizes ranging from $100 \times 100$ to $1000 \times 1000$ in steps of 100 . The dashed curve shows the dependence of $\left\langle R^{2}\right\rangle$ on energy for the largest system size.

To study the localization length exponent $\nu$, we performed extensive calculations of the mean extension $\langle R\rangle$ of orbits at various energies $E$ around $E_{c}$ and various system sizes. We took only localized trajectories to get rid of uncontrolled surface effects. For the largest system $(3000 \times 3000)$ we were able to study the behavior at energies as low as 0.005 .

In Fig. 2 we show all data calculated at a number of energies and system sizes. For those energies at which the localization length does not exceed the size of the system, the averaged extension of the trajectory increases with decreasing energy toward the percolation level. For energies close enough to the percolation level, the localization length exceeds the system size, and $\langle R\rangle$ saturates. From scaling assumptions we expect that the value of $\langle R\rangle$ at the percolation level should scale with the size of the system $M$ with the same exponent. We have checked this to be really the case. From the data shown in Fig. 2, we have estimated $\nu=1.30 \pm 0.10$. This value again is compatible with that expected from percolation theory ${ }^{15} \nu=\frac{4}{3}$, and agrees with a previous classical calculation for QHE. ${ }^{14}$

\section{CONDUCTIVITY}

Imagine a very large rectangular network of dimensions $N \times M$. Let $N$ be the length of its top (and bottom) row, and $M$ its width. If we connect the top and the bottom row of the network to metallic electrodes, we can measure a current $I$ flowing across the network. The position of the Fermi level inside the electrodes controls the energy of an electron injected into the network. We assume that the applied voltage $U$ is sufficiently small, and does not change the energies and their distribution inside the system. ${ }^{18}$

From Ohm's law we expect the conductance $I / U$ of the system to be proportional to its length $N$ and inversely proportional to its width $M$, so we can define the conductivity $\sigma$ in usual way:

$$
\frac{I}{U}=\sigma \frac{N}{M}
$$

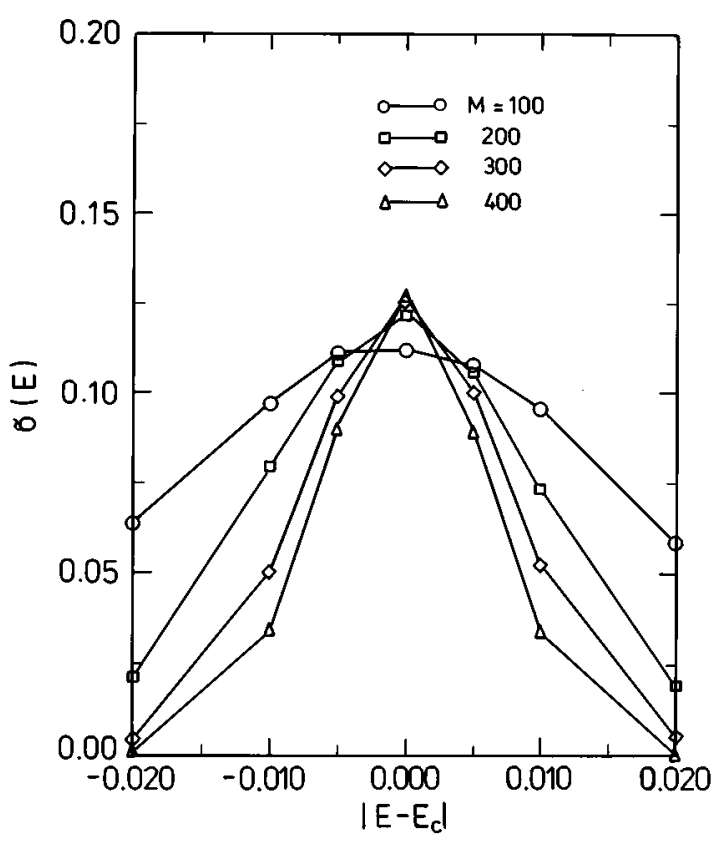

FIG. 3. Energy dependence of the longitudinal conductivity $\sigma_{x x}$ (for a discussion of units see text) for a number of system sizes. The data have been obtained by averaging over 50000 realizations of the disorder. The aspect ratio was taken to be 1 .

In a finite system the current $I$ as well as the conductivity $\sigma$ will depend on the width $M$. The system will be conducting $(I \neq 0)$ at energy $E$, provided there exists a state extending between the electrodes at this energy. Again, in a finite system this will happen for a range of energies $E$ for which $M<\xi(E)$. The conductivity is proportional to the total current. Contrary to the usual situation in percolation problems, ${ }^{15,16}$ there is no difficulty here in calculating total currents across the system, as there are no closed loops on the trajectories connecting both electrodes.

For fixed $N$ the increase of $M$ at a given value of energy leads to a diminishing of the current and also of the longitudinal conductivity which eventually vanishes if $M>\xi(E)$. We have used Eq. (5) to calculate the $E$ - and $M$-dependent conductivity $\sigma$. We have obtained a roughly linear decrease of $\sigma$, calculated at the percolation energy $E_{c}$, with $M$

$$
\sigma_{M}\left(E_{c}\right) \propto M^{-1} \text { for const } N \text {. }
$$

The situation is quite different if we go to the thermodynamic limit by letting $N \rightarrow \infty$ and $M \rightarrow \infty$, but with an aspect ratio $a_{r}=N / M=$ const. The dependence on $E$ and $M$ of the resulting conductivity is shown in Fig. 3. One can see that presumably $\sigma_{M}(E) \rightarrow 0$ with $M, N \rightarrow \infty$ for all $E$ except $E=E_{c}$, where it saturates at large $M$ (and for $a_{r}$ being constant). Thus in the thermodynamic limit we expect a nonzero value of the conductivity exactly at the percolation threshold.

To understand this behavior, and in particular the nonzero value of the conductivity at the percolation level, let us note the differences between our case and the classical percolation. In classical case conductivity is a continous function of probability $p$ of permitted sites, ${ }^{15}$ and vanishes at $p=p_{c}$. It takes nonzero values for $p>p_{c}$, and in particular for $p=1$. In our case the parameter controlling the distance from the 


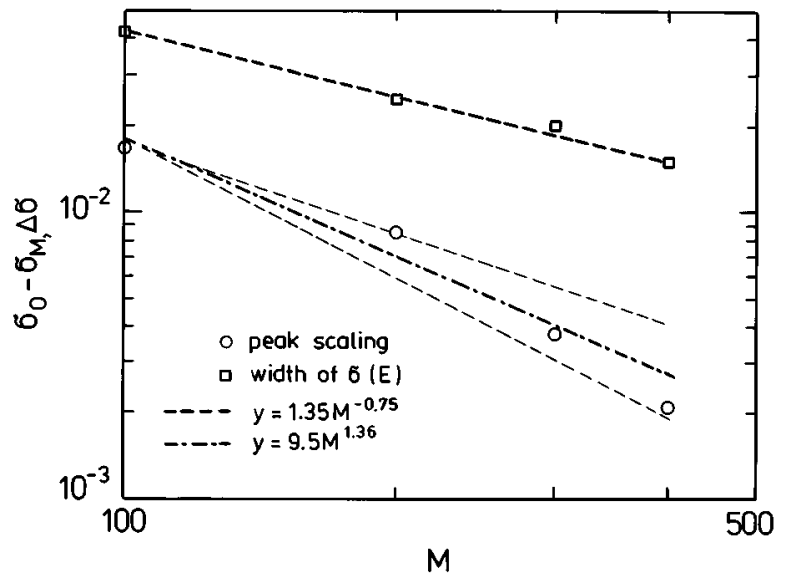

FIG. 4. Analysis of the data shown in Fig. 3. The width of the conductivity scales with an exponent $\eta=1 / \nu=0.75$. The scaling of the peak value is compatible with the previously found value $\eta^{\prime}=1.62$.

percolation level is the position of the Fermi level. Not all properties of the classical percolation systems viewed as functions of $p$ can be traced back here by replacing $p$ by $E$. In particular the region of nonzero values of conductivity in classical percolation ${ }^{15} p_{c}<p \leqslant 1$ shrinks here to a point $E=E_{c}$.

Inspection of Eq. (3) and Fig. (1) shows that diffusion (for $E=E_{c}$ ) over closed trajectories is normal, $k=1$, while the particle motion over open trajectories is superdiffusive with $k=1.2$. It is this regime which leads to nonzero value for the conductivity at the percolation level.

Another way to understand this is to realize that the particle motion considered in our work takes place on the hulls of the clusters. They are fractal objects with fractal dimension $d_{f}$ [see Eq. (4)]. There are a few important differences between classical diffusion and diffusion on fractals, ${ }^{19}$ but we shall not discuss them here. Moreover, within the subspace of open trajectories the particles do not diffuse but propagate in a single direction only, which leads to $k>1$.

The approach to the thermodynamic limit can be studied by looking at the decrease of the width in energy $(\Delta E)_{\sigma}$ of the conductivity with increasing system size. We show this dependence in Fig. 4 on a log-log plot. The width of $\sigma(E)$ vanishes (as it should) with the inverse of classical localization length exponent $\nu$.

In view of a recent proposal, ${ }^{20}$ it is also interesting to study the scaling of the peak value of the conductivity, i.e., the dependence of $\sigma\left(E_{c}\right)$ on $M$. In the present approach this quantity does not behave statistically very well. The data shown in Fig. 3 have been averaged over 50000 realizations of disorder, and close inspection still shows sizable fluctuations.

If, however, we take the changes with $M$ of $\sigma_{M}\left(E_{c}\right)$ seriously, then the quantity ${ }^{20}$

$$
\Delta \sigma_{M}\left(E_{c}\right)=\sigma_{\infty}-\sigma_{M}\left(E_{c}\right)=\alpha M^{-\eta^{\prime}}
$$

( $\sigma_{\infty}$ denotes the conductivity in the thermodynamic limit) vanishes with the exponent $\eta^{\prime}=1.36 \pm 0.3$. This number does not exclude the value $\eta^{\prime}=1.63 \pm 0.03$ found in previous fully quantum-mechanical studies of the QHE by means of direct numerical evaluation of the Kubo formula for $\sigma_{x x}{ }^{20}$ This exponent has previously been identified with the fractal dimension of the wave function $D(2)$. In Ref. 20 it has been also found that the value of $\sigma_{\infty}$ was universal, and equal to $0.5 e^{2} / h$. To convert our arbitrary units of Fig. 3 to the physical ones, Büttiker's ${ }^{21}$ approach in the limit of ideal contacts can, in principle, be used with due attention to the boundary conditions. We have, however, an independent method of calibration for the conductivity axis (and finding the value of $\left.\sigma_{\infty}\right)$ which is free of boundary conditions problems. To this end it is enough to use diffusion constant $D$ determined from time simulations (see Fig. 1) and Einstein relation

$$
\sigma_{x x}=e^{2} \rho D,
$$

where $\rho$ is the density of states. Similar arguments as presented previously ${ }^{14}$ lead to the simple result $\rho=1 / h$. From the data presented in Fig. 1 , we have $D \approx 0.49 \pm 0.02$, and $\sigma_{\infty}=(0.49 \pm 0.02)\left(e^{2} / h\right)$.

The quality of our results for the scaling of width of conductivity and its approach of the limiting (seemingly universal) value and their analysis is shown in Fig. 4. If the identification of $\eta$ and $D(2)$ is correct, we can expect, at least in principle, a scaling of the peak of "classical" conductivity $\sigma_{x x}$ with the fractal dimension $d_{f}$ which we have just estimated to be $1.70 \pm 0.05$. This number is certainly larger than the most optimistic estimate of $\eta^{\prime}$, and our classical estimation of this exponent compares better with the quantum value than with the expected classical one. The obtained agreement is a little bit surprising, because in our classical treatment all quantities scale with known classical exponents. This reflects some internal consistency in our approach. Is the agreement of our exponent for the scaling of the conductivity peak with a quantum one just a lucky coincidence, or does it mean that the peak value of the conductivity scales with an exponent which does not depend on the approach, or do they not differ much? We think this point deserves further studies.

\section{SUMMARY AND DISCUSSION}

We applied classical methods to analyze the behavior of the network model, which is the proper model to study the quantum Hall system in long-range random potentials. All the exponents we calculated except $\eta^{\prime}$, which describes the scaling of the conductivity peak, agree with the expected values for a classical two-dimensional percolating system. ${ }^{15}$ It has been found that the classical treatment of the network model gives a reasonable overall description of the properties of the system it models. The critical exponents, however, are all classical ones, and differ from those found in quantum-mechanical treatments. The fact that the width of the energy-dependent conductivity scales with the same exponent as the independently determined localization length is an indication of the internal consistency of the approach. As already mentioned, the surprising similarity of the value of the exponent $\eta^{\prime}$ found here and in quantum mechanical calculations, is not clear at present, and further studies are needed to clarify this point.

We have also tried to simulate the quantum tunneling in the present approach. To this end at each saddle point where 
an electron arrived we have calculated the transmission probability by using the formula ${ }^{17}$

$$
T(E)=\left\{1+\exp \left[2 \pi\left(E-E_{s}\right) / V^{\prime \prime} l^{2}\right]\right\}^{-1},
$$

where $V^{\prime \prime}=\left(V_{x}^{\prime \prime} V_{y}^{\prime \prime}\right)^{1 / 2}$ and $V_{x}^{\prime \prime}$ and $V_{y}^{\prime \prime}$ are curvatures at the saddle point along the principal directions. The characteristic value of $V^{\prime \prime}$ is $\Gamma / d^{2}$. $\Gamma$ here is the characteristic disorderinduced broadening of the Landau level, and $d$ is the correlation radius of the random potential. In actual calculations we have estimated $V^{\prime \prime}=\Gamma / d^{2}$. Assuming that $\Gamma=0.5$ and $d / l=3$, we end up with $2 \pi / V^{\prime \prime} l^{2}=100$ for the numerical value of the coefficient multiplying the energy in the exponent. Our preliminary results are inconclusive as yet. At the time being, we have not been able to show that $\left\langle R^{2}(t)\right\rangle$ saturates in the limit $t \rightarrow \infty$. Consequently it is not clear whether our model with tunneling included is critical at all. The calculated correlation length exponent $\nu$ has become energy dependent taking on larger values for energies closer to the percolation energy $E_{c}$. A qualitative understanding of this result is rather easy. With tunneling included, each particle orbiting the closed trajectory has a chance (presumably after long time) to hop onto the neighboring trajectory. This should lead to a small increase of the conductivity. Our results are suprising in the respect that we obtained large increases of $\left\langle R^{2}\right\rangle$ and $\sigma(E)$ when allowing for tunneling at about $5 \%$ of all saddle points. One reason might be that our observation time ( $10^{6}$ steps) is still too small, or that our method of accepting the tunneling event by comparing the tunneling probability with an additional random number may be not sufficient. An absence of a phase transition from localized to delocalized trajectories would possibly have to be interpreted as an indication that not only the tunneling alone but also backscattering effects (interference) do play an important role in driving the system into the correct universality class. ${ }^{22}$ This, however, means that the proposed theory, ${ }^{8}$ based on classical percolation supplemented by tunneling, does not give a proper explanation of the localizationdelocalization transition.

The other possibility is that the values of the correlation length exponents are not universal and, in a noninteracting system, do depend on the width of the saddle-point distribution (cf. the two values found in Ref. 10 for two values of $W$ ), or at constant distribution width (as in our case) on the system size. This point of view is certainly a non-orthodox one. But there are additional arguments which may be of some relevance to this discussion. A recent result, claimed to be exact, from renormalization group-calculations ${ }^{24}$ for the case of a white-noise random potential states that $\nu=2$. There exist some arguments ${ }^{25}$ that the network model with no (phase) randomness should show $\nu=1$. To conclude this paragraph: there recently appeared a number of results which render the necessity of better understanding the delocalization transition in QHE.

The network model has recently also been applied ${ }^{23}$ to a description of spin degenerate systems for which experiments measure values of the localization exponent half large as for transitions between spin-split Landau levels. In the present approach at a given energy $E$ there would be two electrons, one with spin-up and another with spin-down simultaneously "walking" through the network. If their energy differs by, say, $\Delta_{1 / 2}$, their trajectories (though corresponding to the same energy $E$ ) will belong to two different equipotential lines. In an extremal case the trajectory of one of the electrons can span the (finite) system, while the other can be localized inside it. The coupling between both trajectories should, however, be taken into account, which means that the larger orbits will make the main contribution to the studied quantity. ${ }^{26}$ This in turn means that in our approach some averaging over an energy bin $\Delta_{1 / 2}$ would be necessary to obtain the values of the exponent valid for a spindegenerate band. This subject is beyond the scope of the present work.

\section{ACKNOWLEDGMENTS}

We thank Dr. Berndt Gammel for most helpful discussions and comments. One of us (K.I.W.) acknowledges the warm hospitality extended to him during a stay at TU München. His visit there was possible thanks to Grant No. HCM-6419 from the European Community.
* On leave of absence from Institute of Physics, M. CurieSkłodowska University, Radziszewskiego 10a, PL- $20031 \mathrm{Lu}-$ blin, Poland. Electronic address: karol@tytan.umcs.lublin.pl

†Present address: Department of Physics and Materials Science Institute, University of Oregon, Eugene, OR 97403. Electronic address: fevers@darkwing.uoregon.edu

${ }^{1}$ M. Janßen, O. Viehweger, U. Fastenrath, and J. Hajdu, in Introduction to the Theory of the Integer Quantum Hall Effect, edited by Jànos Hajdu (VCH, Weinheim, 1994).

${ }^{2}$ The Quantum Hall Effect, edited by R. Prange and S. M. Girvin (Springer Verlag, Berlin, 1986).

${ }^{3}$ K. von Klitzing, G. Dorda, and M. Pepper, Phys. Rev. Lett. 45, 494 (1980); K. von Klitzing, Rev. Mod. Phys. 58, 519 (1980).

${ }^{4}$ S. Koch, R. J. Haug, K. von Klitzing, and K. Ploog, Phys. Rev. Lett. 67, 883 (1991).

${ }^{5}$ H. P. Wei, D. C. Tsui, M. A. Paalanen, and A. M. M. Pruisken, Phys. Rev. Lett. 61, 1294 (1988)

${ }^{6}$ A. M. M. Pruisken, Phys. Rev. Lett. 61, 1294 (1988).

${ }^{7}$ B. Huckestein and B. Kramer, Phys. Rev. Lett. 64, 1437 (1990);
H. Aoki and T. Ando, J. Phys. Soc. Jpn. 54, 2239 (1985); S.Hikami, Prog. Theor. Phys. 76, 1210 (1986).

${ }^{8}$ G. V. Mil'nikov and I. M. Sokolov, Pis'ma Zh. Éksp. Teor. Fiz. 48, 494 (1988) [JETP Lett. 48, 536 (1988)]

${ }^{9}$ J. T. Chalker and P. D. Coddington, J. Phys. C 21, 2665 (1988).

${ }^{10}$ Alex Hansen and C. A. Lütgen, Phys. Rev. B 51, 5566 (1995).

${ }^{11}$ Dung-Hai Lee, Ziqiang Wang, and Steven Kivelson, Phys. Rev. Lett. 70, 4130 (1993).

${ }^{12}$ S. V. Iordansky, Solid State Commun. 43, 1 (1982); R. F. Kazarinov and S. Luryi, Phys. Rev. B 25, 7626 (1982); S. A. Trugman, ibid. 277539 (1983).

${ }^{13}$ J. F. G. Eastmond, Ph.D. thesis, University of Oxford, 1992 (unpublished).

${ }^{14}$ F. Evers and W. Brenig, Z. Phys. B 94, 155 (1994).

${ }^{15}$ Dietrich Stauffer and Amnon Aharony, Introduction to Percolation Theory, 2nd ed. (Taylor and Francis, London, 1992).

${ }^{16}$ M. B. Isichenko, Rev. Mod. Phys. 64, 961 (1992).

${ }^{17}$ H. A. Fertig and B. I. Halperin, Phys. Rev. B 36, 7969 (1987).

${ }^{18}$ In reality one has to take the screening into account, which is very 
effective inside the QHE probe. See, e.g., W. Brenig and P. K. Yogeshwar, Europhys. Lett. 7, 737 (1988), and references cited there. Thus our assumption of no influence of external voltages on the distribution of saddle point energies is justified.

${ }^{19}$ S. Havlin and D. Ben-Avraham, Adv. Phys. 36, 695 (1987).

${ }^{20}$ B. Gammel and W. Brenig, Phys. Rev. Lett. 73, 3286 (1994).

${ }^{21}$ M. Büttiker, Phys. Rev. B 38, 9375 (1988).
${ }^{22}$ L. Jaeger, J. Phys. C 3, 2441 (1991).

${ }^{23}$ D. K. K. Lee and J. T. Chalker, Phys. Rev. Lett. 72, 1510 (1994). ${ }^{24}$ L.Moriconi (unpublished).

${ }^{25}$ Andreas W. W. Ludwig, Matthew P. A. Fisher, R. Shankar, and G. Grinstein, Phys. Rev. B 50, 7526 (1994).

${ }^{26}$ D. G. Polyakov and M. E. Raikh (unpublished). 